\title{
A note on functional limit theorems for compound Cox processes*
}

\author{
V. Yu. Korolev ${ }^{\dagger}$ A. V. Chertok
}

\begin{abstract}
An improved version of the functional limit theorem is proved establishing weak convergence of random walks generated by compound doubly stochastic Poisson processes (compound Cox processes) to Lévy processes in the Skorokhod space under more realistic moment conditions. As corollaries, theorems are proved on convergence of random walks with jumps having finite variances to Lévy processes with variance-mean mixed normal distributions, in particular, to stable Lévy processes, generalized hyperbolic and generalized variance-gamma Lévy processes.
\end{abstract}

Key words: stable distribution; normal variance-mean mixture; Lévy process; $\alpha$-stable Lévy process; compound doubly stochastic Poisson process (compound Cox process); Skorokhod space; transfer theorem

\section{Introduction}

In financial mathematics the evolution of (the logarithms of) stock prices and financial indexes on small time horizons is often modeled by random walks. The simplest example of such an approach is the Cox-RossRubinstein model (see, e. g., [29]). At the same time most successful (adequate) models of the dynamics of (the logarithms of) financial indexes on large time horizons are subordinated Wiener processes (processes of Brownian motion with random time such as generalized hyperbolic processes, in particular, variance gamma $(\mathrm{VG})$ processes and normal \\inverse Gaussian (NIG) processes. These models very well describe the observed heavy-tailedness and leptokurticity of the empirical (statistical) distributions of the increments of financial indices and, in particular, of stock prices on comparatively short time intervals.

Functional limit theorems are a quite natural link between random walks and subordinated Wiener processes. The operation of subordination gives a good explanation of the presence of heavy tails in the empirical distributions of the increments of (the logarithms of) stock prices and financial indexes.

In the book [10] and the papers [17, 18] it was proposed to model the evolution of non-homogeneous chaotic stochastic processes, in particular, of the dynamics of stock prices and financial indexes, by random walks generated by compound doubly stochastic Poisson processes (compound Cox pocesses). A doubly stochastic Poisson process (also called a Cox process) is a stochastic point process of the form $N_{1}\left(\Lambda(t)\right.$ ), where $N_{1}(t), t \geq 0$, is a homogeneous Poisson process with unit intensity and the stochastic process $\Lambda(t), t \geq 0$, is independent of $N_{1}(t)$ and possesses the following properties: $\Lambda(0)=0, \mathrm{P}(\Lambda(t)<\infty)=1$ for any $t>0$, the sample paths of $\Lambda(t)$ do not decrease and are right-continuous. A compound Cox process is a random sum of independent identically distributed random variables in which the number of summands follows a Cox process. Similar continuous-time random walks were considered in [13, 14].

This approach, based on the universal principle of non-decrease of entropy in closed systems, was developed in [5, 20, 19. In the paper 27, this approach was successfully applied to modeling the evolution of limit order books in the high-frequency financial trading systems. In the framework of this approach the principal idea is that the moments at which the state of the system under consideration changes form a chaotic point stochastic process on the time axis. Moreover, this point process turns out to be non-stationary (time-non-homogeneous) because the changes of the state of the limit order book are to a great extent subject to the influence of non-stationary information flows. As is known, most reasonable probabilistic models of non-stationary (timenon-homogeneous) chaotic point processes are doubly stochastic Poisson processes also called Cox processes (see, e. g., [12, 5]). These processes are defined as Poisson processes with stochastic intensities. Pure Poisson processes can be regarded as best models of stationary (time-homogeneous) chaotic flows of events [5]. Recall that the attractiveness of a Poisson process as a model of homogeneous discrete stochastic chaos is due to at least two reasons. First, Poisson processes are point processes characterized by that time intervals between

\footnotetext{
*Research supported by Russian Scientific Foundation, project 14-11-00364.

${ }^{\dagger}$ Faculty of Computational Mathematics and Cybernetics, Lomonosov Moscow State University; Institute of Informatics Problems FRC CSC RAS; victoryukorolev@yandex.ru

${ }_{\ddagger}^{\ddagger}$ Faculty of Computational Mathematics and Cybernetics, Lomonosov Moscow State University, Euphoria Group LLC; a.v.chertok@gmail.com

§aculty of Computational Mathematics and Cybernetics, Lomonosov Moscow State University; sasha.korchagin@gmail.com

『Higher School of Economics National Research University, Moscow; e_kossova@mail.ru

" Vologda State University; Institute of Informatics Problems FRC CSC RAS; ISEDT RAS; a_zeifman@mail.ru
} 
successive points are independent random variables with one and the same exponential distribution and, as is well known, the exponential distribution possesses the maximum differential entropy among all absolutely continuous distributions concentrated on the nonnegative half-line with finite expectations, whereas the entropy is a natural and convenient measure of uncertainty. Second, the points forming the Poisson process are uniformly distributed along the time axis in the sense that for any finite time interval $\left[t_{1}, t_{2}\right], t_{1}<t_{2}$, the conditional joint distribution of the points of the Poisson process which fall into the interval $\left[t_{1}, t_{2}\right]$ under the condition that the number of such points is fixed and equals, say, $n$, coincides with the joint distribution of the order statistics constructed from an independent sample of size $n$ from the uniform distribution on $\left[t_{1}, t_{2}\right]$ whereas the uniform distribution possesses the maximum differential entropy among all absolutely continuous distributions concentrated on finite intervals and very well corresponds to the conventional impression of an absolutely unpredictable random variable (see, e. g., [10, 5]).

In 24 some functional limit theorems were proved establishing convergence of random walks generated by compound Cox processes with jumps possessing finite variances to Lévy processes with symmetric distributions including symmetric strictly stable Lévy processes. In the paper [27] these results were extended to a nonsymmetric case and applied to modeling the evolution of the order flow imbalance process, an integral characteristic of the behavior of the limit order book.

The present paper presents a further development of the models and techniques proposed in our previous papers [24, 27]. In this paper we improve and generalize the results of the mentioned papers by relaxing the conditions and correcting some inaccuracies. Our presentation essentially relies on the techniques developed in [24].

The paper is organized as follows. Section 2 contains some basic material on the Skorokhod space, stable distributions and Lévy processes. In section 3 we prove general functional limit theorem establishing the conditions for convergence of compound Cox processes to Lévy processes in the Skorokhod space in terms of the behavior of the cumulative intensities of Cox processes. For this purpose we extend the classical results presented, say, in [15]. In Section 4 we consider the conditions for the convergence of compound Cox processes with elementary jumps possessing finite variances to the Lévy processes with variance-mean mixed normal one-dimensional distributions, that is, to subordinated Wiener processes.

\section{Skorokhod space. Lévy processes}

Let $D=D[0,1]$ be the space of real functions defined on $[0,1]$, right-continuous and having finite left-side limits.

Let $\mathcal{F}$ be the class of strictly increasing mappings of $[0,1]$ onto itself. Let $f$ be a non-decreasing function on $[0,1]$ with $f(0)=0, f(1)=1$. Set $\|f\|=\sup _{s \neq t}|\log [(f(t)-f(s)) /(t-s)]|$. If $\|f\|<\infty$, then the function $f$ is continuous and strictly increasing and, hence, belongs to $\mathcal{F}$.

Define the distance $d_{0}(x, y)$ in the set $D[0,1]$ as the greatest lower bound of the set of positive numbers $\epsilon$, for which $\mathcal{F}$ contains a function $f$ such that $\|f\| \leqslant \epsilon$ and $\sup _{t}|x(t)-y(f(t))| \leqslant \epsilon$.

It can be shown that the space $D[0,1]$ is complete with respect to the distance $d_{0}$. The metric space $\mathcal{D}=\left(D[0,1], d_{0}\right)$ is called the Skorokhod space. Everywhere in what follows we will consider stochastic processes as $\mathcal{D}$-valued random elements.

Let $X, X_{1}, X_{2}, \ldots$ be $\mathcal{D}$-valued random elements. Let $T_{X}$ be a subset of $[0,1]$ such that $0 \in T_{X}, 1 \in T_{X}$ and if $0<t<1$, then $t \in T_{X}$ if and only if $\mathrm{P}(X(t) \neq X(t-))=0$. The following theorem establishing sufficient conditions for the weak convergence of stochastic processes in $\mathcal{D}$ (denoted below as $\Longrightarrow$ and assumed as $n \rightarrow \infty$ ) is well-known.

Theorem A. Let $\left(X_{n}\left(t_{1}\right), \ldots, X_{n}\left(t_{k}\right)\right) \Longrightarrow\left(X\left(t_{1}\right), \ldots, X\left(t_{k}\right)\right)$ for any natural $k$ and $t_{1}, \ldots, t_{k}$ belonging to $T_{X}$. Let $\mathrm{P}(X(1) \neq X(1-))=0$ and let there exist a non-decreasing continuous function $F$ on $[0,1]$, such that for any $\epsilon>0$

$$
\mathrm{P}\left(\left|X_{n}(t)-X_{n}\left(t_{1}\right)\right| \geqslant \epsilon,\left|X_{n}\left(t_{2}\right)-X_{n}(t)\right| \geqslant \epsilon\right) \leqslant \epsilon^{-2 \nu}\left[F\left(t_{2}\right)-F\left(t_{1}\right)\right]^{2 \gamma}
$$

for $t_{1} \leqslant t \leqslant t_{2}$ and $n \geqslant 1$, where $\nu \geqslant 0, \gamma>1 / 2$. Then $X_{n} \Longrightarrow X$.

The PROOF of Theorem A can be found, for example, in [7.

Everywhere in what follows the symbol $\stackrel{d}{=}$ stands for the coincidence of distributions.

By a Lévy process we will mean a stochastic process $X(t), t \geqslant 0$, possessing the following properties: (i) $X(0)=0$ almost surely; (ii) $X(t)$ is the process with independent increments, that is, for any $N \geqslant 1$ and $t_{0}, t_{1}, \ldots, t_{N}\left(0 \leqslant t_{0} \leqslant t_{1} \leqslant \ldots \leqslant t_{N}\right)$ the random variables $X\left(t_{0}\right), X\left(t_{1}\right)-X\left(t_{0}\right), \ldots, X\left(t_{N}\right)-X\left(t_{N-1}\right)$ are jointly independent; (iii) $X(t)$ is a homogeneous process, that is, $X(t+h)-X(t) \stackrel{d}{=} X(s+h)-X(s)$ for any $s, t, h>0$; (iv) the process $X(t)$ is stochastically continuous, that is, for any $t \geqslant 0$ and $\epsilon>0 \lim _{s \rightarrow t} \mathrm{P}(|X(t)-X(s)|>\epsilon)=0$; (v) sample paths of the process $X(t)$ are right-continuous and have finite left-side limits. 
Denote the characteristic function of the random variable $X(t)$ as $\psi_{t}(s)\left(\psi_{t}(s)=\mathrm{E} e^{i s X(t)}, s \in \mathbb{R}\right)$. The following statement describes a well-known property of Lévy processes.

Lemma 1. Let $X=X(t), t \geqslant 0$, be a Lévy process. For any $t>0$ the characteristic function of the random variable $X(t)$ is infinitely divisible and has the form

$$
\psi_{t}(s)=\left[\psi_{1}(s)\right]^{t}=\left[\mathrm{E} e^{i s X(1)}\right]^{t}, \quad s \in \mathbb{R} .
$$

Conversely, let $Y$ be an arbitrary infinitely divisible random variable. Then the family of infinitely divisible distributions with characteristic functions of the form $\left[\mathrm{E} e^{i s Y}\right]^{t}$ completely determines finite-dimensional distributions of a Lévy process $X(t), t \geqslant 0$, moreover, $X(1) \stackrel{d}{=} Y$.

The properties of Lévy processes are described in detail in [6, 28,

By $G_{\alpha, \theta}(x)$ we will denote the distribution function of the strictly stable law with the characteristic exponent $\alpha$ and parameter $\theta$ corresponding to the characteristic function $\mathfrak{g}_{\alpha, \theta}(s)=\exp \left\{-|s|^{\alpha} \exp \left\{-\frac{i}{2} \pi \theta \alpha \operatorname{sign} s\right\}\right\}, s \in \mathbb{R}$, where $0<\alpha \leqslant 2,|\theta| \leqslant \theta_{\alpha}=\min \left\{1, \frac{2}{\alpha}-1\right\}$. To symmetric strictly stable distributions there corresponds the value $\theta=0$. To one-sided stable distributions there correspond the values $\theta=1$ and $0<\alpha \leqslant 1$.

In what follows any random variable with the distribution function $G_{\alpha, \theta}(x), 0<\alpha<2$, will be denoted $Z_{\alpha, \theta}$. It is well known that $\mathrm{E}\left|Z_{\alpha, \theta}\right|^{\delta}<\infty$ for any $\delta \in(0, \alpha)$, but the moments of orders higher or equal to $\alpha$ of the random variable $Z_{\alpha, \theta}$ do not exist (see, e. g., 31]).

The distribution function of the standard normal law $(\alpha=2, \theta=0)$ will be denoted $\Phi(x), \Phi(x)=$ $\int_{-\infty}^{x} \varphi(z) d z, \varphi(x)=\frac{1}{\sqrt{2 \pi}} e^{-x^{2} / 2}$.

It is well known that the distribution function $G_{\alpha, 0}(x)$ of the symmetric strictly stable law can be represented as a scale mixture of normal laws:

$$
G_{\alpha, 0}(x)=\int_{0}^{\infty} \Phi\left(\frac{x}{\sqrt{u}}\right) d G_{\alpha / 2,1}(u), \quad x \in \mathbb{R}
$$

(see, e.g., 31, Theorem 3.3.1). To this representation there corresponds the following relation in terms of characteristic functions:

$$
\mathfrak{g}_{\alpha, 0}(s)=\int_{0}^{\infty} \exp \left\{-\frac{s^{2} u}{2}\right\} d G_{\alpha / 2,1}(u), \quad s \in \mathbb{R} .
$$

A Lévy process $X(t), t \geqslant 0$, will be called $\alpha$-stable, if $\mathrm{P}(X(1)<x)=G_{\alpha, \theta}(x), x \in \mathbb{R}$. It can be shown (see, e.g., [8]) that if $X(t), t \geqslant 0$, is a Lévy process, then $X(t)$ is $\alpha$-stable if and only if

$$
X(t) \stackrel{d}{=} t^{1 / \alpha} X(1), \quad t \geqslant 0
$$

\section{Convergence of compound Cox processes to Lévy processes}

In what follows without noticeable loss of generality we will consider stochastic processes defined for $0 \leqslant t \leqslant 1$. Actually, this means that we consider the behavior of compound Cox processes on finite time horizons. The equality of the right bound of the horizon to one can be achieved by an appropriate choice of the units of measurement of time. In other words, we will concentrate on studying the case of the Skorokhod space $\mathcal{D}$.

In order to introduce reasonable asymptotics which formalizes the condition of «infinite» growth of intensities of the flows of informative events, and makes it possible to construct asymptotic («heavy-traffic») approximations to Cox processes, fix a time instant $t$ and introduce an auxiliary parameter $n$. Everywhere in what follows the convergence will be meant as $n \rightarrow \infty$ unless otherwise specified. So, consider a sequence of compound Cox processes of the form

$$
Q_{n}(t)=\sum_{i=1}^{N_{1}^{(n)}\left(\Lambda_{n}(t)\right)} X_{n, i}, \quad t \geqslant 0
$$

where $\left\{N_{1}^{(n)}(t), t \geqslant 0\right\}_{n \geqslant 1}$ is a sequence of Poisson processes with unit intensities; for each $n=1,2, \ldots$ the random variables $X_{n, 1}, X_{n, 2}, \ldots$ are identically distributed; for any $n \geqslant 1$ the random variables $X_{n, 1}, X_{n, 2}, \ldots$ and the process $N_{1}^{(n)}(t), t \geqslant 0$, are independent; for each $n=1,2, \ldots \Lambda_{n}(t), t \geqslant 0$, is a subordinator, that is, a non-decreasing positive Lévy process, independent of the process

$$
Z_{n}(t)=\sum_{i=1}^{N_{1}^{(n)}(t)} X_{n, i}, \quad t \geqslant 0
$$


and such that $\Lambda_{n}(0)=0$.

Assume that there exist $\delta \in(0,1], \delta_{1} \geqslant \frac{1}{2}$ and the constants $C_{n} \in(0, \infty)$ providing for all $t \in(0,1]$ the validity of the inequality

$$
\mathrm{E} \Lambda_{n}^{\delta}(t) \leqslant\left(C_{n} t\right)^{\delta_{1}}
$$

For example, assume that $\Lambda_{n}(t)$ is a stable Lévy process, that is, $\mathrm{P}\left(\Lambda_{n}(1)<x\right)=G_{\alpha, 1}(x)$ with some $0<\alpha<1$. Then for any $\rho \in(0, \alpha) \subseteq(0,1]$ we have $\mathrm{E} \Lambda_{n}^{\rho}(1)<\infty$ and, moreover, in accordance with (3) we have $\mathrm{E} \Lambda_{n}^{\rho}(t)=t^{\rho / \alpha} \mathrm{E} \Lambda_{n}^{\rho}(1)$, that is, condition (6) holds for any $\delta \in[\alpha / 2, \alpha)$ with $\delta_{1}=\delta / \alpha \in\left[\frac{1}{2}, 1\right)$ and $C_{n}=\left(\mathrm{E} \Lambda_{n}^{\delta}(1)\right)^{1 / \alpha}$.

Assume that

$$
0<m_{n}^{\beta} \equiv \mathrm{E}\left|X_{n, 1}\right|^{\beta}<\infty .
$$

for some $\beta \in(0,1]$.

Everywhere in what follows for definiteness we assume that $\sum_{i=1}^{0}=0$.

From (4) and (5) it is easy to see that $Q_{n}(t)=Z_{n}\left(\Lambda_{n}(t)\right)$. Since for each $n \geqslant 1$ both $Z_{n}(t)$ and $\Lambda_{n}(t)$ are independent Lévy processes, and, moreover, $\Lambda_{n}(t)$ is a subordinator, then the superposition $Q_{n}(t)=Z_{n}\left(\Lambda_{n}(t)\right)$ is also a Lévy process (see, e. g., Theorem 3.1.1 in [16). Hence the following statement follows.

Lemma 2. For any $0 \leqslant t_{1}<t_{2}<\infty$ and any $n \geqslant 1$ we have $Q_{n}\left(t_{2}\right)-Q_{n}\left(t_{1}\right) \stackrel{d}{=} Q_{n}\left(t_{2}-t_{1}\right)$.

Lemma 3. Let $Q_{n}(t)$ be a compound Cox process (4) satisfying conditions (6) and (7). Then for any $t \in[0,1]$ and any $\epsilon>0$ we have $\mathrm{P}\left(\left|Q_{n}(t)\right| \geqslant \epsilon\right) \leqslant\left(\epsilon^{-\beta} m_{n}^{\beta}\right)^{\delta} \cdot\left(C_{n} t\right)^{\delta_{1}}$.

Proof. Since one-dimensional distributions of the Cox process (4) are mixed Poisson, we have

$$
\begin{gathered}
\mathrm{P}\left(\left|Q_{n}(t)\right| \geqslant \epsilon\right)=\mathrm{P}\left(\left|\sum_{j=1}^{N_{1}^{(n)}\left(\Lambda_{n}(t)\right)} X_{n, j}\right| \geqslant \epsilon\right)=\sum_{k=0}^{\infty} \mathrm{P}\left(N_{1}^{(n)}\left(\Lambda_{n}(t)\right)=k\right) \mathrm{P}\left(\left|\sum_{j=1}^{k} X_{n, j}\right| \geqslant \epsilon\right)= \\
=\int_{0}^{\infty}\left[\sum_{k=0}^{\infty} e^{-\lambda} \frac{\lambda^{k}}{k !} \mathrm{P}\left(\left|\sum_{j=1}^{k} X_{n, j}\right| \geqslant \epsilon\right)\right] d \mathrm{P}\left(\Lambda_{n}(t)<\lambda\right) .
\end{gathered}
$$

The change of the order of summation and integration is possible due to the obvious uniform convergence of the series. Continue (8) by the sequential application of the Markov and Jensen inequalities with $\delta \in(0,1]$ taking part in $(6)$ and $\beta \in(0,1]$ taking part in (7). As a result we obtain

$$
\begin{gathered}
\mathrm{P}\left(\left|Q_{n}(t)\right| \geqslant \epsilon\right) \leqslant \frac{1}{\epsilon^{\beta \delta}} \int_{0}^{\infty}\left[\sum_{k=0}^{\infty} e^{-\lambda} \frac{\lambda^{k}}{k !} \mathrm{E}\left|\sum_{j=1}^{k} X_{n, j}\right|^{\beta \delta}\right] d \mathrm{P}\left(\Lambda_{n}(t)<\lambda\right) \leqslant \\
\leqslant \frac{1}{\epsilon^{\beta \delta}} \int_{0}^{\infty}\left[\sum_{k=0}^{\infty} e^{-\lambda} \frac{\lambda^{k}}{k !}\left(\mathrm{E}\left|\sum_{j=1}^{k} X_{n, j}\right|^{\beta}\right)^{\delta}\right] d \mathrm{P}\left(\Lambda_{n}(t)<\lambda\right),
\end{gathered}
$$

since with $\delta \in(0,1]$ the function $f(x)=x^{\delta}$ is concave for $x \geqslant 0$. It is easy to see that $\mathrm{E}\left|\sum_{j=1}^{k} X_{n, j}\right|^{\beta} \leqslant$ $\sum_{j=1}^{k} \mathrm{E}\left|X_{n, j}\right|^{\beta}=k m_{n}^{\beta}$ for $0<\beta \leqslant 1$. Therefore, continuing (9) with the account of the Jensen inequality for concave functions and (6), we obtain

$$
\begin{aligned}
& \mathrm{P}\left(\left|Q_{n}(t)\right| \geqslant \epsilon\right) \leqslant \frac{\left(m_{n}^{\beta}\right)^{\delta}}{\epsilon^{\beta \delta}} \int_{0}^{\infty}\left(\sum_{k=0}^{\infty} e^{-\lambda} \frac{k^{\delta} \lambda^{k}}{k !}\right) d \mathrm{P}\left(\Lambda_{n}(t)<\lambda\right)=\frac{\left(m_{n}^{\beta}\right)^{\delta}}{\epsilon^{\beta \delta}} \int_{0}^{\infty} \mathrm{E}\left[N_{1}^{(1)}(\lambda)\right]^{\delta} d \mathrm{P}\left(\Lambda_{n}(t)<\lambda\right) \leqslant \\
\leqslant & \frac{\left(m_{n}^{\beta}\right)^{\delta}}{\epsilon^{\beta \delta}} \int_{0}^{\infty}\left[\mathrm{E} N_{1}^{(1)}(\lambda)\right]^{\delta} d \mathrm{P}\left(\Lambda_{n}(t)<\lambda\right)=\frac{\left(m_{n}^{\beta}\right)^{\delta}}{\epsilon^{\beta \delta}} \int_{0}^{\infty} \lambda^{\delta} d \mathrm{P}\left(\Lambda_{n}(t)<\lambda\right)=\frac{\left(m_{n}^{\beta}\right)^{\delta}}{\epsilon^{\beta \delta}} \cdot \mathrm{E} \Lambda_{n}^{\delta}(t) \leqslant \frac{\left(m_{n}^{\beta}\right)^{\delta}}{\epsilon^{\beta \delta}} \cdot\left(C_{n} t\right)^{\delta_{1}} .
\end{aligned}
$$

The lemma is proved.

To establish weak convergence of the stochastic processes $Q_{n}(t)$ in the Skorokhod space $\mathcal{D}$, first it is required to find the limit distribution of the random variables $Q_{n}(t)$ for each $t>0$. The symbol $\stackrel{d}{\longrightarrow}$ will denote convergence in distribution, that is, pointwise convergence of the distribution functions in all continuity points of the limit distribution function.

Let $t=1$. Denote $N_{n}=N_{1}^{(n)}\left(\Lambda_{n}(1)\right)$. Assume that for some $k_{n} \in \mathbb{N}$ the convergence

$$
\mathrm{P}\left(X_{n, 1}+\ldots+X_{n, k_{n}}<x\right) \stackrel{d}{\longrightarrow} H(x)
$$

takes place, where $H(x)$ is some infinitely divisible distribution function. 
Also assume that

$$
\mathrm{P}\left(\Lambda_{n}(1)<k_{n} x\right) \stackrel{d}{\longrightarrow} \mathrm{P}(U<x),
$$

where $U$ is a nonnegative random variable such that its distribution is not degenerate in zero. Notice that since $\Lambda_{n}(t)$ is a Lévy process, then the random variable $U$ is infinitely divisible being the weak limit of infinitely divisible random variables.

Lemma 4. Let $N_{n}=N_{1}^{(n)}\left(\Lambda_{n}\right), n \geqslant 1$, where $\left\{N_{1}^{(n)(t)}, t \geqslant 0\right\}, n=1,2, \ldots$ are standard Poisson processes and $\Lambda_{n}, n=1,2, \ldots$ are positive random variables such that for each $n \geqslant 1$ the random variable $\Lambda_{n}$ is independent of the process $N_{1}^{(n)}(t)$. Then $\mathrm{P}\left(N_{n}<k_{n} x\right) \stackrel{d}{\longrightarrow} A(x)$ for some infinitely increasing sequence $k_{n}$ of real numbers and some distribution function $A(x)$ if and only if $\mathrm{P}\left(\Lambda_{n}<k_{n} x\right) \stackrel{d}{\longrightarrow} A(x)$.

For the proof see [10].

From Lemma 4 it follows that convergence (11) is equivalent to

$$
\mathrm{P}\left(N_{n}<k_{n} x\right) \stackrel{d}{\longrightarrow} \mathrm{P}(U<x) .
$$

By the Gnedenko-Fahim transfer theorem [9] conditions (10) and (12) imply that

$$
Q_{n}(1)=X_{n, 1}+\ldots+X_{n, N_{n}} \stackrel{d}{\longrightarrow} Q,
$$

where $Q$ is a random variable with the characteristic function $\mathfrak{f}(s)=\int_{0}^{\infty}(h(s))^{u} d \mathrm{P}(U<u), h(s)$ being the characteristic function corresponding to the distribution function $H(x)$. Note that the distribution function $H(x)$ may not satisfy the condition $H(-x)=1-H(x)$ for all $x \geqslant 0$, that is, it may not be symmetric.

Let $Y$ be an infinitely divisible random variable with the distribution function $H(x)$. Since both $Y$ and $U$ are infinitely divisible, we can define independent Lévy processes $Y(t)$ and $U(t), t \geqslant 0$, such that $Y(1) \stackrel{d}{=} Y$ and $U(1) \stackrel{d}{=} Y$. Then with the account of Lemma 1 it is easy to verify that $\mathfrak{f}(s)=\mathrm{E} e^{i s Q}=\operatorname{E} \exp \{i s Y(U(1))\}$, $s \in \mathbb{R}$, that is, $Q \stackrel{d}{=} Y(U(1))$. Moreover, repeating the reasoning from [16] (see Theorem 3.3.1 there), we can easily see that the random variable $Q$ is infinitely divisible and hence, we can define a Lévy process $Q(t), t \geqslant 0$, such that $Q(1) \stackrel{d}{=} Q$. From Lemma 1 and the abovesaid it follows that we can regard $Q(t)$ as the superposition: $Q(t) \stackrel{d}{=} Y(U(t))$.

Since according to (13) we have $Q_{n}(1)=\sum_{i=1}^{N_{n}} X_{n, i} \Longrightarrow Q(1)$, and both $Q_{n}(t)$ and $Q(t)$ are Lévy processes, then, using (2) we can conclude that for any $t>0$

$$
Q_{n}(t)=\sum_{i=1}^{N_{n, 1}\left(\Lambda_{n}(t)\right)} X_{n, i} \stackrel{d}{\longrightarrow} Q(t) .
$$

Since the processes $Q_{n}(t)$ and $Q(t), 0 \leqslant t \leqslant 1$, are Lévy processes, then almost all their sample paths belong to the Skorokhod space $\mathcal{D}$.

Consider the question what additional conditions are required to provide the weak convergence of the compound Cox process $Q_{n}(t)$ to the Lévy process $Q(t)$ in the space $\mathcal{D}$. We will consider each of the conditions of Theorem A one by one.

First, without loss of generality, let $0 \leqslant t_{1}<t_{2}<\ldots<t_{k} \leqslant 1$. The convergence $\left(Q_{n}\left(t_{1}\right), \ldots, Q_{n}\left(t_{k}\right)\right) \stackrel{d}{\longrightarrow}$ $\left(Q\left(t_{1}\right), \ldots, Q\left(t_{k}\right)\right)$ is equivalent to the convergence

$$
\begin{gathered}
\left(Q_{n}\left(t_{1}\right), Q_{n}\left(t_{2}\right)-Q_{n}\left(t_{1}\right), \ldots, Q_{n}\left(t_{k}\right)-Q_{n}\left(t_{k-1}\right)\right) \stackrel{d}{\longrightarrow} \\
\stackrel{d}{\longrightarrow}\left(Q\left(t_{1}\right), Q\left(t_{2}\right)-Q\left(t_{1}\right), \ldots, Q\left(t_{k}\right)-Q\left(t_{k-1}\right)\right),
\end{gathered}
$$

since the linear transform $\left(x_{1}, x_{2}, \ldots, x_{k-1}, x_{k}\right) \longmapsto\left(x_{1}, x_{2}-x_{1}, \ldots, x_{k}-x_{k-1}\right)$ of $\mathbb{R}^{k}$ to $\mathbb{R}^{k}$ is one-to-one and continuous in both directions. But convergence (15) follows from (14) and the fact that both $Q_{n}(t)$ and $Q(t)$ are Lévy processes.

Second, we have to check the condition $\mathrm{P}(Q(1) \neq Q(1-))=0$. This condition holds if and only if $\lim _{t \rightarrow 1-} \mathrm{P}(|Q(1)-Q(t)|>\epsilon)=0$ for any $\epsilon>0$ (see relation (15.16) in [7). Consider $\mathrm{P}(|Q(1)-Q(t)|>\epsilon)$. Since $Q(t)$ is a Lévy process, then $Q(1)-Q(t) \stackrel{d}{=} Q(1-t)$ by Lemma 2. Therefore, $\mathrm{P}(|Q(1)-Q(t)|>\epsilon)=$ $\mathrm{P}(|Q(1-t)|>\epsilon)$. For each $\epsilon>0$ and each $t \in[0,1]$ there exists an $\epsilon_{t} \in[\epsilon / 2, \epsilon]$ such that the points $\pm \epsilon_{t}$ are continuity points of the distribution function of the random variable $Q(1-t)$. Since $Q_{n}(t) \stackrel{d}{\longrightarrow} Q(t)$ for each $t \in[0,1]$, then $\mathrm{P}\left(|Q(1-t)|>\epsilon_{t}\right)=\lim _{n \rightarrow \infty} \mathrm{P}\left(\left|Q_{n}(1-t)\right|>\epsilon_{t}\right)$. Thus, for any $\epsilon>0$ and any $t \in[0,1]$ we have

$$
\mathrm{P}(|Q(1-t)|>\epsilon) \leqslant \mathrm{P}\left(|Q(1-t)|>\epsilon_{t}\right)=\lim _{n \rightarrow \infty} \mathrm{P}\left(\left|Q_{n}(1-t)\right|>\epsilon_{t}\right) \leqslant \limsup _{n \rightarrow \infty} \mathrm{P}\left(\left|Q_{n}(1-t)\right|>\epsilon_{t}\right) .
$$


Continuing (16) with the account of (6) and applying Lemma 3 , for $\delta \in(0,1]$ taking part in (6) we obtain

$$
\begin{gathered}
\mathrm{P}(|Q(1-t)|>\epsilon) \leqslant \limsup _{n \rightarrow \infty} \mathrm{P}\left(\left|Q_{n}(1-t)\right|>\epsilon_{t}\right) \leqslant \\
\leqslant \limsup _{n \rightarrow \infty}\left(\epsilon_{t}^{-\beta} m_{n}^{\beta}\right)^{\delta}\left(C_{n}|1-t|\right)^{\delta_{1}} \leqslant\left((2 / \epsilon)^{\beta \delta}|1-t|\right)^{\delta_{1}} \limsup _{n \rightarrow \infty}\left(m_{n}^{\beta}\right)^{\delta} C_{n}^{\delta_{1}} .
\end{gathered}
$$

Therefore, if

$$
K \equiv \limsup _{n \rightarrow \infty} C_{n}^{\delta_{1} / \delta} m_{n}^{\beta}<\infty
$$

then (17) implies

$$
\lim _{t \rightarrow 1-} \mathrm{P}(|Q(1)-Q(t)|>\epsilon) \leqslant 4\left(K \epsilon^{-\beta}\right)^{\delta} \lim _{t \rightarrow 1-}|1-t|^{\delta_{1}}=0 .
$$

Third, check condition (1) under the assumption that (6) and (18) hold. As it has been noted above, $Q_{n}(t)$ is a Lévy process and hence, it has independent increments. Therefore,

$$
\mathrm{P}\left(\left|Q_{n}(t)-Q_{n}\left(t_{1}\right)\right| \geqslant \epsilon,\left|Q_{n}\left(t_{2}\right)-Q_{n}(t)\right| \geqslant \epsilon\right)=\mathrm{P}\left(\left|Q_{n}(t)-Q_{n}\left(t_{1}\right)\right| \geqslant \epsilon\right) \cdot \mathrm{P}\left(\left|Q_{n}\left(t_{2}\right)-Q_{n}(t)\right| \geqslant \epsilon\right) .
$$

Consider the first multiplier on the right-hand side of (19). By Lemma $2, Q_{n}(t)-Q_{n}\left(t_{1}\right) \stackrel{d}{=} Q_{n}\left(t-t_{1}\right)$. With the account of (18), by Lemma 3 we obtain

$$
\mathrm{P}\left(\left|Q_{n}(t)-Q_{n}\left(t_{1}\right)\right| \geqslant \epsilon\right)=\mathrm{P}\left(\left|Q_{n}\left(t-t_{1}\right)\right| \geqslant \epsilon\right) \leqslant\left(K \epsilon^{-\beta}\right)^{\delta}\left|t-t_{1}\right|^{\delta_{1}} .
$$

For the second multiplier on the right-hand side of (19) we similarly obtain

$$
\mathrm{P}\left(\left|Q_{n}\left(t_{2}\right)-Q_{n}(t)\right| \geqslant \epsilon\right)=\mathrm{P}\left(\left|Q_{n}\left(t_{2}-t\right)\right| \geqslant \epsilon\right) \leqslant\left(K \epsilon^{-\beta}\right)^{\delta}\left|t_{2}-t\right|^{\delta_{1}} .
$$

Thus, from (20) and (21) it follows that

$$
\mathrm{P}\left(\left|Q_{n}(t)-Q_{n}\left(t_{1}\right)\right| \geqslant \epsilon,\left|Q_{n}\left(t_{2}\right)-Q_{n}(t)\right| \geqslant \epsilon\right) \leqslant\left(K \epsilon^{-\beta}\right)^{2 \delta}\left[\left(t-t_{1}\right)\left(t_{2}-t\right)\right]^{\delta_{1}}
$$

It is easy to see that for any $t_{1} \leqslant t \leqslant t_{2}$ we have $\left(t-t_{1}\right)\left(t_{2}-t\right) \leqslant \frac{1}{4}\left(t_{2}-t_{1}\right)^{2}$. Substituting this estimate in (22) we obtain $\mathrm{P}\left(\left|Q_{n}(t)-Q_{n}\left(t_{1}\right)\right| \geqslant \epsilon,\left|Q_{n}\left(t_{2}\right)-Q_{n}(t)\right| \geqslant \epsilon\right) \leqslant \epsilon^{-2 \beta \delta}\left[\frac{1}{2} K\left(t_{2}-t_{1}\right)\right]^{2 \delta_{1}}$. Therefore, if conditions (6) and (18) hold, then condition (1) holds with $F(t) \equiv \frac{1}{2} K t, \nu=\beta \delta$ and $\gamma=\delta_{1}$.

Summarizing this reasoning related to checking the conditions of Theorem A, we arrive at the following statement.

Theorem 1. Assume that the random variables $\left\{X_{n, j}\right\}_{j \geqslant 1}, n=1,2, \ldots$, (the jumps of the compound Cox process $Q_{n}(t)$, see (4)), satisfy conditions (10) with some $k_{n} \in \mathbb{N}$ and (7) with some $\beta \in(0,1]$. Let the processes $Q_{n}(t)$ be lead by non-decreasing positive Lévy processes $\Lambda_{n}(t)$ satisfying conditions $(6)$ with some $\delta \in(0,1]$, $\delta_{1} \geqslant \frac{1}{2}$ and (11) with the same $k_{n}$. Also assume that condition (18) holds. Then the processes $Q_{n}(t)$ weakly converge in the Skorokhod space $\mathcal{D}$ to the Lévy process $Q(t)$ such that

$$
\mathrm{E} \exp \{i s Q(1)\}=\int_{0}^{\infty}(h(s))^{u} d \mathrm{P}(U<u), \quad s \in \mathbb{R},
$$

where $h(s)$ is the characteristic function corresponding to the distribution function $H(x)$ in (10).

It is worth noting that actually Theorem 1 deals with the well-studied weak convergence of special semimartingales with stationary increments, see, e. g., [15]. However, the superposition-type structure of the processes considered in the present paper makes it possible to relax the conditions required in the general case, say, in Corollary VII.3.6 of [15] where it is assumed that (in our terminology) $\delta=\delta_{1}=1$. Moreover, in [24] and some subsequent papers a more restrictive condition was used instead of (18).

From Theorem 1 it obviously follows that if in (10) $H(x)=G_{\alpha, \theta}(x)$ for some admissible $\alpha \in(0,2)$ and $\theta \in[-1,1]$ and the processes $\Lambda_{n}(t)$ are asymptotically degenerate, that is, in (11) $\mathrm{P}(U=1)=1$, then in (23) $\mathrm{E} \exp \{i s Q(1)\}=\mathfrak{g}_{\alpha, \theta}(s)$, that is, the limiting process is the stable Lévy process.

However, in 24] it was demonstrated that stable Lévy processes can appear as limits for compound Cox processes even when the variances of elementary increments of a compound Cox process are finite. To generalize this result, consider the following corollary of Theorem 1 .

Corollary 1. Assume that the random variables $\left\{X_{n, j}\right\}_{j} \geqslant 1, n=1,2, \ldots$, satisfy condition (10) with some $k_{n} \in \mathbb{N}$ and $H(x)=G_{\alpha, 0}(x)$ for some $\alpha \in(0,2]$ so that condition $(7)$ holds for any $\beta \in(0, \alpha) \bigcap(0,1]$. Let the compound Cox processes $Q_{n}(t)$ be lead by non-decreasing positive Lévy processes $\Lambda_{n}(t)$ satisfying conditions 
(11) with the same $k_{n}$ and $\mathrm{P}(U<x)=G_{\alpha^{\prime}, 1}(x)$ for some $\alpha^{\prime} \in(0,1]$ so that condition $(6)$ holds with some $\delta \in$ $\left[\alpha^{\prime} / 2, \alpha^{\prime}\right)$ and $\delta_{1}=\delta / \alpha^{\prime} \in\left[\frac{1}{2}, 1\right)$. Also assume that condition (18) holds for some $\beta \in(0, \alpha)$. Then the processes $Q_{n}(t)$ weakly converge in the Skorokhod space $\mathcal{D}$ to the Lévy process $Q(t)$ such that $\mathrm{P}(Q(1)<x)=G_{\alpha \alpha^{\prime}, 0}(x)$, $x \in \mathbb{R}$. Proof. To prove this result is suffices to notice that in the case under consideration $\mathfrak{f}_{\alpha, 0}(s)=e^{-|s|^{\alpha}}$, $s \in \mathbb{R}$, so that in $(23)$

$$
\mathrm{E} \exp \{i s Q(1)\}=\int_{0}^{\infty} e^{-u|s|^{\alpha}} d G_{\alpha^{\prime}, 1}(u)=\int_{0}^{\infty} e^{-\left|s u^{1 / \alpha}\right|^{\alpha}} d G_{\alpha^{\prime}, 1}(u)=\mathrm{E} \exp \left\{i s Z_{\alpha, 0} Z_{\alpha^{\prime}, 1}^{1 / \alpha}\right\}, \quad s \in \mathbb{R}
$$

where the random variables $Z_{\alpha, 0}$ and $Z_{\alpha^{\prime}, 1}$ are independent. But from Theorem 3.3.1 in [31] it follows that $Z_{\alpha, 0} Z_{\alpha^{\prime}, 1}^{1 / \alpha} \stackrel{d}{=} Z_{\alpha \alpha^{\prime}, 0}$. The corollary is proved.

In turn, the result from [24] mentioned above follows from Corollary 1, if $\alpha=2$. This case corresponds to the situation in which the variances of the summands (elementary jumps) are assumed finite. As it has been already said, in most applied problems there are no reasons to reject this assumption. Therefore in what follows we will concentrate our attention on the case where the elementary increments of the compound Cox process have finite variances and consider the conditions of convergence of compound Cox processes to some popular models, in particular, to Lévy processes with variance-mean mixed normal one-dimensional distributions such as generalized hyperbolic Lévy processes or generalized variance-gamma Lévy processes.

\section{Lévy processes with variance-mean mixed normal distributions as asymptotic approximations to compound Cox processes}

Denote $a_{n}=\mathrm{E} X_{n, 1}$ and $\sigma_{n}^{2}=\mathrm{D} X_{n, 1}$. From the classical theory of limit theorems it is well known that if, as $n \rightarrow \infty$, the conditions

$$
k_{n} a_{n} \longrightarrow a, \quad k_{n} \sigma_{n}^{2} \longrightarrow \sigma^{2} \text { and } k_{n} \mathrm{E}\left(X_{n, 1}-a_{n}\right)^{2} \mathbb{I}\left(\left|X_{n, 1}-a_{n}\right| \geqslant \epsilon\right) \longrightarrow 0
$$

hold for some $a \in \mathbb{R}, 0<\sigma^{2}<\infty$ and any $\epsilon>0$, then convergence (10) takes place with $H(x) \equiv \Phi\left(\sigma^{-1}(x-a)\right)$. In this case the distribution function $F(x)$ of the limit random variable $Q(1)$ in Theorem 1 is a variance-mean mixture of normal laws. Recently it was demonstrated that normal variance-mean mixtures appear as limiting in simple limit theorems for random sums of independent identically distributed random variables [21. Namely, let $\left\{\xi_{n, j}\right\}_{j \geqslant 1}, n=1,2, \ldots$, be a double array of row-wise (for each fixed $n$ ) independent and identically distributed random variables. Let $\left\{\nu_{n}\right\}_{n} \geqslant 1$ be a sequence of integer nonnegative random variables such that for each $n \geqslant 1$ the random variables $\nu_{n}, \xi_{n, 1}, \xi_{n, 2}, \ldots$ are independent. Denote $S_{n, k}=\xi_{n, 1}+\ldots+\xi_{n, k}$. The following theorem was proved in [21].

TheOREM B. Assume that there exist: a sequence $\left\{k_{n}\right\}_{n \geqslant 1}$ of natural numbers and finite numbers $\alpha \in \mathbb{R}$ and $\sigma>0$ such that

$$
\mathrm{P}\left(S_{n, k_{n}}<x\right) \stackrel{d}{\longrightarrow} \Phi\left(\frac{x-\alpha}{\sigma}\right) .
$$

Assume that $\nu_{n} \rightarrow \infty$ in probability. Then the distribution functions of random sums $S_{n, \nu_{n}}$ converge to some distribution function $F(x): \mathrm{P}\left(S_{n, \nu_{n}}<x\right) \stackrel{d}{\longrightarrow} F(x)$, if and only if there exists a distribution function $A(x)$ such that

$$
A(0)=0, \quad F(x)=\int_{0}^{\infty} \Phi\left(\frac{x-\alpha z}{\sigma \sqrt{z}}\right) d A(z),
$$

and $\mathrm{P}\left(\nu_{n}<x k_{n}\right) \stackrel{d}{\longrightarrow} A(x)$.

Theorem B and Lemma 3 yield the following result.

Theorem 2. Assume that the random variables $\left\{X_{n, j}\right\}_{j \geqslant 1}, n=1,2, \ldots$, the jumps of the compound Cox process $Q_{n}(t)$, see (4)) possess finite variances and satisfy conditions (24) with some $k_{n} \in \mathbb{N}, a \in \mathbb{R}$ and $\sigma>0$. Let the the processes $Q_{n}(t)$ be lead by non-decreasing positive Lévy processes $\Lambda_{n}(t)$ satisfying condition (6) with some $\delta \in(0,1], \delta_{1} \geqslant \frac{1}{2}$. Also assume that

$$
K \equiv \limsup _{n \rightarrow \infty} C_{n}^{\delta_{1} / \delta}\left(\sigma_{n}+\left|a_{n}\right|\right)<\infty .
$$

Then the processes $Q_{n}(t)$ weakly converge in the Skorokhod space $\mathcal{D}$ to a Lévy process $Q(t)$ if and only if there exists a nonnegative random variable $U$ such that

$$
\mathrm{P}(Q(1)<x)=\int_{0}^{\infty} \Phi\left(\frac{x-a u}{\sigma \sqrt{u}}\right) d \mathrm{P}(U<u), \quad x \in \mathbb{R}
$$


and condition (11) holds with the same $k_{n}$.

Proof. It suffices to take $\beta=1$ in the proof of lemma 3 and notice that $m_{n}^{1} \leqslant \sigma_{n}+\left|a_{n}\right|$ so that condition (26) can play the role of (18).

The class of distributions of form (27) was systematically considered by O. Barndorff-Nielsen and his colleagues [2, 3, 4, in order to introduce generalized hyperbolic distributions and study their properties.

The class of normal variance-mean mixtures (27) is very wide. For example, it contains generalized hyperbolic laws with generalized inverse Gaussian mixing distributions [1, 2], generalized variance gamma (GVG) distributions with generalized gamma mixing distributions [22, 23], symmetric strictly stable laws with strictly stable mixing distributions concentrated on the positive half-line, generalized exponential power distributions, and many other types.

Generalized hyperbolic distributions demonstrate exceptionally high adequacy when they are used to describe statistical regularities in the behavior of characteristics of various complex open systems, in particular, turbulent systems and financial markets. There are dozens of dozens of publications dealing with models based on generalized hyperbolic distributions. Recently it was discovered that generalized variance gamma distributions demonstrate even better fit to empirical data. Therefore below we will concentrate our attention on functional limit theorems establishing the convergence of compound Cox processes to generalized hyperbolic Lévy processes and generalized variance gamma Lévy processes yielding the possibility of the use of such processes as convenient «heavy-traffic» asymptotic approximations.

Denote the density of the generalized inverse Gaussian distribution by $p_{G I G}(x ; \nu, \mu, \lambda)$,

$$
p_{G I G}(x ; \nu, \mu, \lambda)=\frac{\lambda^{\nu / 2}}{2 \mu^{\nu / 2} K_{\nu}(\sqrt{\mu \lambda})} \cdot x^{\nu-1} \cdot \exp \left\{-\frac{1}{2}\left(\frac{\mu}{x}+\lambda x\right)\right\}, \quad x>0 .
$$

Here $\mu>0, \lambda \geqslant 0$ if $\nu<0 ; \mu>0, \lambda>0$ if $\nu=0$ and $\mu \geqslant 0, \lambda>0$ if $\nu>0, K_{\nu}(z)$ is the modified Bessel function of the third kind with index $\nu, K_{\nu}(z)=\frac{1}{2} \int_{0}^{\infty} y^{\nu-1} \exp \left\{-\frac{z}{2}\left(y+\frac{1}{y}\right)\right\} d y, z \in \mathbb{C}, \operatorname{Re} z>0$. The corresponding distribution function will be denoted $P_{G I G}(x ; \nu, \mu, \lambda)$.

The class of generalized inverse Gaussian distributions is rather rich and contains, in particular, both distributions with exponentially decreasing tails (gamma-distribution $(\mu=0, \nu>0)$ ), and distributions whose tails demonstrate power-type behavior (inverse gamma-distribution $(\lambda=0, \nu<0)$, inverse Gaussian distribution $\left(\nu=-\frac{1}{2}\right)$ and its limit case as $\lambda \rightarrow 0$, the Lévy distribution (the stable distribution $G_{\frac{1}{2}, 1}(x)$ with the characteristic exponent equal to $\frac{1}{2}$ and concentrated on the nonnegative half-line, the distribution of the time for the standard Wiener process to hit the unit level)).

In 1977-78 O. Barndorff-Nielsen [1, 2] introduced the class of generalized hyperbolic distributions as the class of special normal variance-mean mixtures. For convenience, we will use a somewhat simpler parametrization. Let $\alpha \in \mathbb{R}, \sigma>0$. If the generalized hyperbolic distribution function with parameters $\alpha, \sigma, \nu, \mu, \lambda$ is denoted $P_{G H}(x ; \alpha, \sigma, \nu, \mu, \lambda)$, then by definition,

$$
P_{G H}(x ; \alpha, \sigma, \nu, \mu, \lambda)=\int_{0}^{\infty} \Phi\left(\frac{x-\alpha z}{\sigma \sqrt{z}}\right) p_{G I G}(z ; \nu, \mu, \lambda) d z, \quad x \in \mathbb{R} .
$$

Note that in (28) mixing is carried out simultaneously by both location and scale parameters, but since these parameters are directly linked in (28), then actually (28) is a one-parameter mixture.

It is well-known that all generalized hyperbolic distributions are infinitely divisible [1, 2.

From Theorem 2, Corollary 1 and Theorem B with the account of the equivalence of relations (11) and (12) we easily obtain the following result on the convergence of compound Cox processes to generalized hyperbolic Lévy processes.

Corollary 2. Assume that the random variables $\left\{X_{n, j}\right\}_{j} \geqslant 1, n=1,2, \ldots$, the jumps of the compound Cox process $Q_{n}(t)$, see (4)) possess finite variances and satisfy conditions (24) with some $k_{n} \in \mathbb{N}, a \in \mathbb{R}$ and $\sigma>0$. Let the the processes $Q_{n}(t)$ be lead by non-decreasing positive Lévy processes $\Lambda_{n}(t)$ satisfying condition (6) with some $\delta \in(0,1], \delta_{1} \geqslant \frac{1}{2}$. Also assume that condition (26) holds. Then the processes $Q_{n}(t)$ weakly converge in the Skorokhod space $\mathcal{D}$ to a generalized hyperbolic Lévy process $Q(t)$ such that $\mathrm{P}(Q(1)<x)=P_{G H}(x ; a, \sigma, \nu, \mu, \lambda)$ if and only if $\mathrm{P}\left(\Lambda_{n}(1)<k_{n} x\right) \stackrel{d}{\longrightarrow} P_{G I G}(x ; \nu, \mu, \lambda)$ with the same $k_{n}, \nu, \mu$ and $\lambda$.

The class of generalized gamma (GG) distributions was introduced in the paper [30]. Any representative of this class is defined by the probability density

$$
p_{G G}(x ; \nu, \kappa, \delta)=\frac{|\nu|}{\delta^{k \nu} \Gamma(\kappa)} x^{\kappa \nu-1} \exp \left\{-\left(\frac{x}{\delta}\right)^{\nu}\right\}, \quad x \geqslant 0,
$$


where the parameters $\nu \in \mathbb{R}, \kappa, \delta \in \mathbb{R}^{+}$respectively determine the power, shape and scale of the generalized gamma distribution. Here $\Gamma(\kappa)$ is Euler's gamma-function.

The family of GG-distributions contains practically all most popular absolutely continuous distributions concentrated on $\mathbb{R}^{+}$. In particular, this class contains (i) the gamma-distribution $(\nu=1)$, (ii) the exponential distribution $(\nu=1, \kappa=1)$, (iii) the Erlang distribution $(\nu=1, \kappa \in \mathbb{N})$, (iv) the chi-square distribution $(\nu=1, \delta=2)$, (v) the Nakagami distribution $(\nu=2)$, (vi) the half-normal distribution (the distribution of the absolute value of the standard normal random variable) $\left(\nu=2, \kappa=\frac{1}{2}\right)$, (vii) the Rayleigh distribution $(\nu=2, \kappa=1)$, (viii) the chi-distribution $(\nu=2, \delta=\sqrt{2})$, (ix) the Maxwell distribution $(\nu=2, \kappa=3 / 2)$, (x) the Weibull-Gnedenko distribution $(\kappa=1)$, (xi) the inverse gamma-distribution $(\nu=-1)$, (xii) the Lévy distribution, (xiii) the lognormal distribution $(\kappa \rightarrow \infty)$.

Unlike generalized inverse Gaussian laws, generalized gamma laws contain the distributions with exponential power type of decrease of the tail where the posiltive exponent power may be arbitrary.

In 22] the family of generalized variance gamma (GVG) distributions was introduced as the family of normal variance-mean mixtures of the form

$$
P_{G V G}(x ; a, \sigma, \nu, \kappa, \delta)=\int_{0}^{\infty} \Phi\left(\frac{x-a u}{\sigma \sqrt{u}}\right) p_{G G}(u ; \nu, \kappa, \delta) d u, \quad x \in \mathbb{R},
$$

Both classes, GH and GVG distributions, contain (a) symmetric and non-symmetric (skew) Student distributions (including Cauchy distribution), to which in (32) there correspond inverse gamma mixing distributions; (b) variance gamma (VG) distributions) (including symmetric and non-symmetric Laplace distributions), to which in (32) there correspond gamma mixing distributions; (c) normal \\inverse Gaussian (NIG) distributions to which in (32) there correspond inverse Gaussian mixing distributions. However, the class of GVG laws includes normal mixtures with tails decreasing as $e^{-|x|^{\nu}}$ with $0<\nu<1$, which are not included in GH-distributions. This type of tail behavior is of great practical interest.

Not all of GVG distributions are infinitely divisible. But as concerns the important case of Weibull-type decreasing tails mentioned above, it turns out that for $\nu \in(0,1]$ these laws are infinitely divisible. Recall that in the notation introduced above the Weibull-Gnedenko distribution corresponds to the GG-density $p_{G G}(x ; \nu, 1, \delta)$.

Lemma 5. If $\nu \leqslant 1$, then the Weibull-Gnedenko distribution is infinitely divisible.

Proof. As was shown in the paper [26], the Weibull-Gnedenko distribution with $\nu \leqslant 1$ is mixed exponential. But in [1] it was proved that all mixed exponential laws are infinitely divisible. The lemma is proved.

According to lemma 5 , if $\nu \leqslant 1$, then a Lévy process $U(t), t \geqslant 0$, can be defined so that $\mathrm{P}(U(1)<x)=$ $P_{G G}(x ; \nu, 1, \delta), x \in \mathbb{R}$. Such a process will be called the Lévy-Weibull process. Let $W(t)$ be a standard Wiener process independent of $U(t)$.

Corollary 3. Assume that the random variables $\left\{X_{n, j}\right\}_{j} \geqslant 1, n=1,2, \ldots$, (the jumps of the compound Cox process $Q_{n}(t)$, see (4)) possess finite variances and satisfy conditions (24) with some $k_{n} \in \mathbb{N}, a=0$ and $\sigma>0$. Let the the processes $Q_{n}(t)$ be lead by non-decreasing positive Lévy processes $\Lambda_{n}(t)$ satisfying condition (6) with some $\delta \in(0,1], \delta_{1} \geqslant \frac{1}{2}$. Also assume that condition (26) holds. Then the processes $Q_{n}(t)$ weakly converge in the Skorokhod space $\mathcal{D}$ to a subordinated Wiener process $W(U(t))$ with the subordinator $U(t)$ being the Lévy-Weibull process with $\nu \leqslant 1$ if and only if $\mathrm{P}\left(\Lambda_{n}(1)<k_{n} x\right) \stackrel{d}{\longrightarrow} P_{G G}(x ; \nu, 1, \delta)$ with the same $k_{n}$.

\section{References}

[1] Barndorff-Nielsen O. E. Exponentially decreasing distributions for the logarithm of particle size // Proc. Roy. Soc. London. Ser. A, 1977. Vol. A(353). P. 401-419.

[2] Barndorff-Nielsen O. E. Hyperbolic distributions and distributions of hyperbolae // Scand. J. Statist., 1978. Vol. 5. P. 151-157.

[3] Barndorff-Nielsen O. E. Models for non-Gaussian variation, with applications to turbulence // Proc. Roy. Soc. London. Ser. A, 1979. Vol. A(368). P. 501-520.

[4] Barndorff-Nielsen O. E., Kent J., Sørensen M. Normal variance-mean mixtures and z-distributions // International Statistical Review, 1982. Vol. 50. No. 2. P. 145-159.

[5] Bening V., Korolev V. Generalized Poisson Models and Their Applications in Insurance and Finance. Utrecht, VSP, 2002. 
[6] Bertoin J. Lévy Processes. Cambridge Tracts in Mathematics, Vol. 121. - Cambridge: Cambridge University Press, 1996.

[7] Billingsley P. Convergence of Probability Measures. - New York: Wiley, 1968.

[8] Embrechts P., Maejima M. Selfsimilar Processes. - Princeton: Princeton University Press, 2002.

[9] Gnedenko B. V., Fahim H. On a transfer theorem // Soviet Math. Dokl., 1969. Vol. 187. No. 1. P. 15-17.

[10] Gnedenko B. V., Korolev V. Yu. Random Summation: Limit Theorems and Applications. - Boka Raton: CRC Press, 1996.

[11] Goldie C. M. A class of infinitely divisible distributions // Math. Proc. Cambridge Philos. Soc., 1967. Vol. 63. P. 1141-1143.

[12] Grandell J. Doubly Stochastic Poisson Processes. Lecture Notes Mathematics, Vol. 529. - BerlinHeidelberg-New York: Springer, 1976.

[13] Granovsky B. L., Zeifman A. I. The decay function of nonhomogeneous birth and death processes, with application to mean-field models // Stochastic Process. Appl., 1997, Vol. 72. P. 105-120.

[14] Huang H., Kercheval A. N. A generalized birth-death stochastic model for high-frequency order book dynamics // Quantitative Finance, 2012. Vol. 12. No. 4. P. 547-557.

[15] Jacod J., Shiryaev A. N.. Limit theorems for stochastic processes. 2nd edition. Volume 288 of Grundlehren der Mathematischen Wissenschaften [Fundamental Principles of Mathematical Sciences]. - Berlin: SpringerVerlag, Berlin, 2003.

[16] Kashcheev D. E. Modeling the Dynamics of Financial Time Series and Evaluation of Derivative Securities. PhD Thesis. - Tver: Tver State University, 2001 (in Russian).

[17] Korolev $V$. Yu. On convergence of the distributions of random sums of independent random variables to stable laws // Theory Probab. Appl., 1997. Vol. 42. No. 4. P. 818-820.

[18] Korolev V. Yu. Asymptotic properties of extrema of compound Cox processes and their application to some problems of financial mathematics // Theory Probab. Appl., 2000. Vol. 45. No. 1. P. 182-194.

[19] Korolev V. Yu. Probabilistic and Statistical Methods For Decomposition of Volatility of Chaotic Processes. - Moscow: Moscow State University Publishing House, 2011 (in Russian).

[20] Korolev V. Yu., Bening V. E., Shorgin S. Ya. Mathematical Foundation of Risk Theory. 2nd ed. - Moscow: FIZMATLIT, 2011 (in Russian).

[21] Korolev V. Yu. Generalized hyperbolic laws as limit distributions for random sums // Theory of Probability and Its Applications, 2013. Vol. 58. No. 1. P. 117--132.

[22] Korolev V. Yu., Sokolov I. A. Skew Student distributions, variance gamma distributions and their generalizations as asymptotic approximations // Informatics and Its Applications, 2012. Vol. 6. No. 1. P. $2-10$.

[23] Zaks L. M., Korolev V. Yu. Generalized variance gamma distributions as limit laws for random sums // Informatics and Its Applications, 2013. Vol. 7. No. 1. P. 105-115.

[24] Korolev V. Yu., Zaks L. M., Zeifman A. I. On convergence of random walks generated by compound Cox processes to Lévy processes // Statistics and Probability Letters, 2013. Vol. 83. No. 10. P. 2432-2438.

[25] Chertok A., Korolev V., Korchagin A. Modeling high-frequency non-homogeneous order flows by compound Cox processes // Journal of Mathematical Sciences, 2015 (to appear). Available at SSRN, January 14, 2014. http://ssrn.com/abstract=2378975.

[26] Korolev V. Yu., Sokolov I. A. On conditions for the convergence of the distributions of extreme order statistics to the Weibull distribution // Informatics and its Applications, 2014. Vol. 8. No. 3. P. 2-10.

[27] Korolev V. Yu, Chertok A. V., Korchagin A. Yu, Zeifman A. I. Modeling high-frequency order flow imbalance by functional limit theorems for two-sided risk processes // Applied Mathematics and Computation, 2015. Vol. 253. P. 224-241 
[28] Sato K. Lévy Processes and Infinitely Divisible Distributions. - Cambridge: Cambridge University Press, 1999.

[29] Shiryaev A. N. Essentials of Stochastic Finance: Facts, Models, Theory. - Singapore: World Scientific. 1999.

[30] Stacy E. W. A generalization of the gamma distribution // Annals of Mathematical Statistics, 1962. Vol. 33. P. 1187-1192.

[31] Zolotarev V. M. One-Dimensional Stable Distributions. - Providence, R.I.: American Mathematical Society, 1986. 